\title{
EDITORIAL
}

\section{Same role and same treatment for airway eosinophilia in asthma and COPD?}

\author{
P. Maestrelli
}

C hronic inflammatory diseases of the airway, such as asthma and chronic obstructive pulmonary disease (COPD), are common and represent a substantial amount of the workload of most respiratory physicians. While medications for asthma provide good control of the disease in the majority of cases [1], pharmacotherapy for COPD is less satisfactory since none of the existing drugs has been shown to have a significant effect on the long-term decline in lung function that is the hallmark of this disease [2]. It is, however, underlined in the international guidelines that COPD is a treatable disease [2]. Current strategy in managing stable COPD includes several individualised interventions directed at different aspects of the disease. Most of these interventions are nonpharmacological, such as smoking cessation to prevent the accelerated loss of lung function, influenza and pneumococcal vaccines to prevent respiratory infections, exercise training to improve exercise tolerance and dyspnoea, nutritional support to avoid weight loss, and long-term administration of oxygen to increase survival in patients with chronic respiratory failure. Pharmacotherapy with long-acting bronchodilators is used to reduce symptoms and exacerbations. The addition of inhaled corticosteroids further reduces the frequency of exacerbations in patients with severe COPD $[3,4]$.

This issue of the European Respiratory Journal features a study by SIVA et al. [5], who focused their intervention on an aspect of COPD that has never been considered before. These investigators tested the hypothesis that treatment with corticosteroids aimed at maintaining sputum eosinophils at $<3 \%$ in COPD patients is associated with a reduction in exacerbations of COPD. A total of 82 patients with moderate-to-very severe COPD were randomised into two groups and the follow-up was extended for 12 months. One group was treated according to conventional British Thoracic Society (BTS) guidelines [6] designed to optimise symptoms (BTS group), and the other was treated according to a protocol that had the additional aim of minimising eosinophilic airway inflammation, which was assessed by using the induced sputum eosinophil count (sputum group). The primary outcome regarded the frequency of exacerbation. Compared with the BTS group, patients in the sputum group experienced fewer severe exacerbations (mean reduction $62 \%, \mathrm{p}=0.037$ ), which were defined as a worsening

\section{STATEMENT OF INTEREST: None declared}

CORRESPONDENCE: P. Maestrelli, Dipartimento di Medicina Ambientale e Sanità Pubblica, Università degli Studi di Padova, via Giustiniani 2, 35128 Padova, Italy. Fax: 390498212566 . Email: piero.maestrelli@unipd.it of respiratory symptoms resulting in the patient being admitted to hospital. Although there was no difference between the groups in the frequency of mild and moderate exacerbations, in the score of symptoms and quality of life, and in post-bronchodilator forced expiratory volume in one second, the reduction of severe exacerbation represents an important achievement of the treatment strategy; in fact, exacerbations contribute to the progression of the disease, increase the risk of mortality and lead to enormous economic costs. Moreover, these results were obtained with average daily doses of inhaled or oral corticosteroid that did not exceed those used in the BTS group. Approximately one fourth of the COPD patients in this study exhibited eosinophilic airway inflammation at baseline (sputum eosinophils $>3 \%$ ). These patients appeared to be more prone to severe exacerbation than patients with $<3 \%$ sputum eosinophils and gained most of the benefit from the strategy of minimising eosinophilic airway inflammation. In fact, compared with corresponding subgroups treated according to BTS guidelines, severe exacerbation in the sputum group was reduced by $88 \%$ in those with sputum eosinophils $>3 \%$, while it was reduced by $42 \%$ in those with less sputum eosinophilia.

Some of the findings of SIVA et al. [5] are not surprising, since there is evidence from studies with a different design that corticosteroid treatment is effective in the prevention of COPD exacerbation and that airway eosinophilia predicts a better response to corticosteroids [1, 7]. However, SIVA et al. [5] provided additional information on the relationship between corticosteroids and eosinophilic airway inflammation in COPD. The strategy of a selected intervention with corticosteroids to modulate eosinophil airway inflammation was associated with a magnitude of reduction in severe exacerbation that was higher $(62 \%)$ than that obtained by KARDOS et al. [4] for moderate and severe exacerbation (35\%) with unselected addition of relatively high-dose inhaled corticosteroids to longacting bronchodilator therapy.

The reasons why eosinophils represent a risk factor for severe COPD exacerbation remain undetermined. It is also unknown whether any COPD patient can exhibit airway eosinophilia at a certain stage of the disease or whether COPD patients with eosinophilia may represent a different asthma-like phenotype of the disease. Further investigations are needed to clarify these issues. The presence of different phenotypes, classified according to the profile of airway inflammatory cells, has also been identified in asthma. However, the presence of a predominant eosinophilc 
inflammation is greater in asthmatic patients than in COPD patients. In fact, it was $\sim 75 \%$ in a study that was not biased by corticosteroid treatment [8]. This group of asthmatics exhibited a better response to corticosteroids compared to asthmatics with airway neutrophilia, as did COPD patients with eosinophilic inflammation.

Overall, COPD is regarded as a disease that, compared to asthma, is relatively resistant to corticosteroids. This observation might be the result of the lower proportion of airway eosinophilia in COPD patients than in asthma patients. Actually, if we compare the amount of corticosteroids necessary to minimise sputum eosinophils in the study of SIVA et al. [5] on COPD and in the study of GREEN et al. [9], who used the same management strategy in asthma, we observe that asthmatic patients needed even higher doses than COPD patients (inhaled beclomethasone dose equivalents $1,705 \pm 189$ versus $976 \pm 51 \mu \mathrm{g}$ per patient per day, and oral prednisolone $3.0 \pm 0.8$ versus $2.0 \pm 0.6 \mathrm{mg}$ per patient per day). It is interesting to note that the major benefit of sputum strategy in asthma was to the number of severe exacerbations and admissions to hospital, suggesting that airway eosinophilia has a similar role in asthma and COPD. The hypothesis that eosinophils in the airway might predict asthma exacerbation is consistent with the results of the Salmeterol or Corticosteroids (SOCS) trial [10]. This study demonstrated that an increase in sputum eosinophil counts in the 2 weeks following discontinuation of inhaled corticosteroids has high sensitivity (90\%) for asthma deterioration occurring over the subsequent 14 weeks.

Taken together, these findings regarding COPD and asthma suggest that the assessment of airway inflammation may aid the achievement of a more efficient use of corticosteroids. With this strategy, corticosteroid treatment is targeted at those individuals who would most benefit from it, which is at variance with the universal use of corticosteroids, according to the level of severity of asthma or COPD, as indicated by current guidelines. This strategy is probably more relevant in COPD than in asthma because the proportion of patients who will benefit from corticosteroids is smaller in the former disease. However, we need to be cautious when evaluating the value of sputum strategy in clinical decision-making in the management of COPD, for the following reasons. First, the study of SIVA et al. [5] was performed on a relatively selected population of COPD patients attending a single specialised centre and the number of patients investigated is rather small compared with the principal multicentre long-term trials of corticosteroid therapy in COPD. Secondly, measurement of sputum eosinophil count is not a current component of routine clinical practice and there are reservations about its feasibility. The induction and analysis of sputum is somewhat complex and its cost/benefit ratio has not yet been determined. Moreover, the success rate of sputum induction, $\sim 80 \%$ (in good hands), is unsatisfactory and limits its clinical utility. The use of exhaled nitric oxide (NO) as a surrogate marker of airway eosinophilia may be helpful in asthma but seems a poor alternative to induced sputum in COPD. This is partly because NO levels are influenced by smoking [11] and partly because exhaled NO measured at a constant mouth flow in the presence of severe airway obstruction is usually underestimated [12]. Finally, $\sim 40 \%$ of the patients in the sputum management group required regular oral corticosteroids to minimise eosinophilic airway inflammation. Given the large body of evidence on sideeffects, long-term treatment with oral corticosteroids is at present not recommended [2]. New prospective studies should demonstrate whether the new sputum strategy will modify this unfavourable benefit/risk ratio.

In summary, eosinophils may be detected in asthmatic and chronic obstructive pulmonary disease airways. However, the proportion of patients with airway eosinophilia is greatly different in the two diseases. Both in asthma and in chronic obstructive pulmonary disease the presence of airway eosinophilia is predictive of a better response to corticosteroid treatment. Most of the benefit of corticosteroids is on severe exacerbation of both diseases, suggesting a similar role of these cells in airway diseases that are, in general, phenotypically dissimilar. These findings provide a rationale for a treatment strategy based on sputum eosinophil count. Other longer prospective studies, including cost/benefit evaluation, are needed before the utility of this strategy in clinical practice can be established.

\section{REFERENCES}

1 Workshop report: Global Initiative for Asthma - updated 2006. www.ginasthma.com/GuidelinesResources.asp?11= $2 \& 12=0$ Date last accessed: February 12, 2007.

2 Workshop report: Global Initiative for Chronic Obstructive Lung Disease - updated 2006. Available from www.who. int/respiratory/copd/GOLD_WR_06.pdf Date last accessed: February 12, 2007.

3 Nannini L, Cates CJ, Lasserson TJ, Poole P. Combined corticosteroid and long acting beta-agonist in one inhaler for chronic obstructive pulmonary disease. Cochrane Database Syst Rev 2004; 3: CD003794.

4 Kardos P, Wencker M, Glaab T, Vogelmeier C. Impact of salmeterol/fluticasone propionate versus salmeterol on exacerbations in severe chronic obstructive pulmonary disease. Am J Respir Crit Care Med 2007; 175: 144-149.

5 Siva R, Green RH, Brightling CE, et al. Eosinophilic airway inflammation and exacerbations of COPD: a randomised controlled trial. Eur Respir J 2007; 29: 906-913.

6 BTS guidelines for the management of COPD. The COPD Guidelines Group of the Standards of Care Committee of the BTS. Thorax 1997; 52: Suppl. 5, s1-s28.

7 Leigh R, Pizzichini MM, Morris MM, Maltais F, Hargreave FE, Pizzichini E. Stable COPD: predicting benefit from high-dose inhaled corticosteroid treatment. Eur Respir J 2006; 27: 964-971.

8 Green RH, Brightling CE, Woltmann G, Parker D, Wardlaw AJ, Pavord ID. Analysis of induced sputum in adults with asthma: identification of subgroup with isolated sputum neutrophilia and poor response to inhaled corticosteroids. Thorax 2002; 57: 875-879.

9 Green RH, Brightling CE, McKenna S, et al. Asthma exacerbations and sputum eosinophil counts: a randomised controlled trial. Lancet 2002; 360: 1715-1721.

10 Deykin A, Lazarus SC, Fahy JV, et al. Sputum eosinophil count predict asthma control after discontinuation of inhaled corticosteroids. J Allergy Clin Immunol 2005; 115: 720-727. 
11 Malinovschi A, Janson C, Holmkvist T, Norback D, Merilainen P, Hogman M. Effect of smoking on exhaled nitric oxide and flow-independent nitric oxide exchange parameters. Eur Respir J 2006; 28: 339-345.
12 de Gouw HWFM, Hendriks J, Woltman AM, Twiss IM, Sterk PJ. Exhaled nitric oxide (NO) is reduced shortly after bronchoconstriction to direct and indirect stimuli in asthma. Am J Respir Crit Care Med 1998; 158: 315-318. 